\title{
BOUNDING THE NUMBER OF GENERATORS FOR A CLASS OF IDEALS IN LOCAL RINGS
}

\author{
Christian Gottlieb \\ Department of Mathematics, University of Stockholm \\ S-106 91 Stockholm, Sweden
}

A well-known property of local (Noetherian) rings of dimension one is that there is, for each such ring, a number which uniformly bounds the number of generators of any ideal in the ring. A proof may be found in [7] (Theorem 1.2 of Chapter 3). In this paper we use the notation $\mu(I)$ for the minimal number of elements needed to generate an ideal $I$ in a local ring $R$. Thus $\mu(I)=$ $\ell(I / \mathfrak{m} I)$, where $\mathfrak{m}$ is the maximal ideal in $R$.

In [2] we proved that, when $R$ is one-dimensional, we actually have $\mu(I) \leq \ell(R /(x))$, where $x$ is any element in $\mathfrak{m}$. Thus $\mu(I)$ is bounded by the least colength of a nonunit in $R$ and this number is of course finite since we may choose $x$ such that $(x)$ is $\mathfrak{m}$-primary.

However, it is obvious from the theory of Hilbert functions that there is no uniform bound on $\mu(I)$ if $\operatorname{dim} R>1$ because then $\mu\left(\mathfrak{m}^{n}\right)$ is, for large $n$, a nonconstant polynomial. To find uniform bounds in rings of dimension higher than one we must thus restrict ourselves to appropriate subclasses of the class of all ideals in the ring. We find a result in this spirit in [7] (Theorem 2.1 of Chapter 3), namely that $\operatorname{dim} R \leq 2$ if and only if there is a uniform bound on $\mu(I)$ for all $I$ such that $\mathfrak{m}$ is not an associated prime ideal of $I$. To prove the "if-part" is quite straightforward. Indeed, if $\operatorname{dim} R>2$, let $\mathfrak{p}$ be a prime ideal of height 2 and use the facts that each power of the maximal ideal $\mathfrak{p}_{\mathfrak{p}}$ of $R_{\mathfrak{p}}$ is the extension of a $\mathfrak{p}$-primary ideal of $R$ and that $\mu(I) \geq \mu\left(I_{\mathfrak{p}}\right)$ for all $I$. 
It will follow as a corollary of our theorem below that if $\operatorname{dim} R \leq 2$ and $\mathfrak{m} \notin$ Ass $I$ then $\mu(I) \leq \ell(R / \mathfrak{q})$ holds for every ideal $\mathfrak{q}$ which is generated by a system of parameters. The purpose of the theorem is to generalize this to higher-dimensional rings. We shall show that $\mu(I) \leq \ell(R / \mathfrak{q})$ if $\operatorname{depth} R / I \geq$ $\operatorname{dim} R-1$, where $\mathfrak{q}$ is any ideal generated by a system of parameters. In order to perform a suitable inductive step we need the following lemma. I owe the idea to [1] (Lemma 1.2), which contains a graded analogue. Also compare Lemma 1 of [9].

Lemma 1. Let $R$ be a local Noetherian ring with maximal ideal $\mathfrak{m}$. Let $x \in \mathfrak{m}$ and let $I$ and $J$ be ideals such that $J \subseteq I \subseteq J+(x)$ and $x$ is a nonzerodivisor on $R / I$. Then $I=J$. In particular it follows that $\mu(I)=\mu(\bar{I})$, where $\bar{I}$ is the image of $I$ in $R /(x)$.

Proof. When proving that $I=J$ we may work modulo $J$ and hence assume that $J=(0)$. Then $I \subseteq(x)$ but $x \notin \mathrm{Z}(R / I)$ so $(I: x)=I$, whence it follows that $I \subseteq x I$ and hence, by Nakayama's Lemma, that $I=(0)$. To prove $\mu(I)=\mu(\bar{I})$ take $x_{1}, x_{2}, \ldots, x_{n} \in I$ such that $\bar{x}_{1}, \bar{x}_{2}, \ldots, \bar{x}_{n}$ form a minimal generating set of $(I+(x)) /(x)$. Put $J=\left(x_{1}, x_{2}, \ldots, x_{n}\right)$. Then $J \subseteq I \subseteq J+(x)$ and hence $J=I$. Thus $\mu(I) \leq \mu(\bar{I})$. But $\mu(I) \geq \mu(\bar{I})$ is obvious.

Theorem 2. Let $R$ be a local Noetherian ring of dimension $d \geq 1$. Let $\mathfrak{m}$ be the maximal ideal in $R$ and let $\mathfrak{q}$ be an ideal generated by a system of parameters. Then $\mu(I) \leq \ell(R / \mathfrak{q})$ holds for all $I$ such that depth $R / I \geq d-1$, i.e. all $I$ such that there is a regular sequence in $R / I$ of length $d-1$.

Proof. We use induction on $d$. If $d=1$ the assertion is that $\mu(I) \leq \ell(R /(x))$ for $x \in \mathfrak{m}$, which was proved in [2] Corollary 2. Suppose $d>1$ and that the theorem is proved for rings of lower dimension than $d$. Let $\mathfrak{p}_{1}, \mathfrak{p}_{2}, \ldots, \mathfrak{p}_{k}$ be the minimal prime ideals in $R$. They are all different from $\mathfrak{m}$, since $\operatorname{dim} R>0$. Let $\mathfrak{p}_{k+1}, \mathfrak{p}_{k+2}, \ldots, \mathfrak{p}_{n}$ be the prime ideals associated to $I$. They are all different from $\mathfrak{m}$, since $\operatorname{depth} R / I>0$. We have thus $\mathfrak{q} \nsubseteq \mathfrak{p}_{i}$ for $i=1,2, \ldots, n$. Using the prime-avoidance theorem ([8] 3.61) we find an element $x \in \mathfrak{q} \backslash \mathfrak{q m}$ such that $x \notin \mathfrak{p}_{1} \cup \mathfrak{p}_{2} \cup \cdots \cup \mathfrak{p}_{n}$. Thus $x$ is a nonzerodivisor on $R / I$ which lies outside the minimal prime ideals of $R$, and moreover $x$ is part of a minimal generating set for $\mathfrak{q}$. Now let $\bar{R}=R /(x)$ and let $\bar{I}$ and $\overline{\mathfrak{q}}$ be the images of $I$ and $\mathfrak{q}$ respectively in $\bar{R}$. It follows that $\operatorname{dim} \bar{R}=d-1$ and $\operatorname{depth} \bar{R} / \bar{I}=\operatorname{depth} R /(I+(x))=\operatorname{depth} R / I-1 \geq d-2$. Further $\mu(\overline{\mathfrak{q}})=d-1$ and hence $\overline{\mathfrak{q}}$ is generated by a system of parameters in $\bar{R}$. By induction we thus obtain $\mu(\bar{I}) \leq \ell(\bar{R} / \overline{\mathfrak{q}})$. But $\mu(I)=\mu(\bar{I})$ by Lemma 1. Clearly $\ell(\bar{R} / \overline{\mathfrak{q}})=\ell(R / \mathfrak{q})$. Thus $\mu(I) \leq \ell(R / \mathfrak{q})$.

The special case of Theorem 2 when $\operatorname{dim} R=2$ is reformulated in the following corollary. 
Corollary 3. Let $R$ be a two-dimensional local Noetherian ring with maximal ideal $\mathfrak{m}$. Let $I$ be an ideal such that $\mathfrak{m} \notin$ Ass $I$. Then $\mu(I) \leq \ell(R / \mathfrak{q})$, where $\mathfrak{q}$ is any ideal generated by a system of parameters.

Returning to Theorem 2 we note that $\operatorname{dim} R / I=d-1$ or $\operatorname{dim} R / I=d$. If $\operatorname{dim} R / I=d-1$ then $R / I$ is Cohen-Macaulay and $I$ is said to be a CohenMacaualy ideal. A special instance of this is when $R$ too is Cohen-Macaulay. Then the condition $\operatorname{dim} R / I=d-1$ is equivalent to height $I=1$. We obtain the following corollary which is also a corollary of Theorem 2.1. of [6] (put $h=1$ in Sally's theorem).

Corollary 4. Let $R$ be a local Cohen-Macaulay ring with maximal ideal $\mathfrak{m}$. Let $I$ be a Cohen-Macaulay ideal in $R$ of height one. Then $\mu(I) \leq e(R)$, the multiplicity of $R$.

Proof. Since the hypotheses of Theorem 2 are satisfied we get $\mu(I) \leq \ell(R / \mathfrak{q})$ for each ideal $\mathfrak{q}$ generated by a system of parameters. Suppose first that $R / \mathfrak{m}$ is infinite. Then we may choose $\mathfrak{q}$ so that $e(R)=e(\mathfrak{q})$ (see [10] viii Theorem 22). Moreover, since $R$ is Cohen-Macaulay, it follows that $e(\mathfrak{q})=$ $\ell(R / \mathfrak{q})$ (see [4] Theorem 17.11). Thus $\mu(I) \leq e(R)$. If $R / \mathfrak{m}$ is finite we use the usual trick of passing to the ring $R(u)=R[u]_{\mathfrak{m}[u]}$. This ring has infinite residue class field and we have $e(R(u))=e(R)$ and $\mu(I(u))=\mu(I)$ for every ideal $I$ in $R$.

Remark. The inequality $\mu(I) \leq \ell(R /(x))$, in the one-dimensional case, may be generalized to $\mu(N) \leq \ell(M / x M)$ where $M$ is an $R$-module and $N$ a submodule of $M$. This result was proved in [3], where we studied the function (of $N) \nu_{x}(N)=\ell(N / x N)$, which was proved to be monotone. Later we have become aware that the inequality $\mu(N) \leq \ell(M / x M)$ also was proved by $\mathrm{H}$. Matsumura in [5].

\section{REFERENCES}

1. A. Geramita, Remarks on the number of generators of some homogeneous ideals, Bull. Sci. Math. (2) 107 (1983) no. 2, 193-207.

2. C. Gottlieb, On generators of ideals in one-dimensional local rings, Communications in Algebra 21 (1993) no. 2, 421-425.

3. C. Gottlieb, An integer-valued function related to the number of generators of modules over local rings of small dimension, Communications in Algebra 21 (1993) no. 11, 4115-4118.

4. H. Matsumura, Commutative ring theory, Cambridge studies in advanced mathematics 8, Cambridge University Press 1992.

5. H. Matsumura, Some new results on numbers of generators of ideals in local rings, Topics in Algebra, Banach Center Publications Volume 26 part 2, PWN-Polish Scientific Publishers 1990, 157-161. 
6. J.D. Sally, Bounds for numbers of generators of Cohen-Maculay ideals, Pacific J. Math. 63 (1976) no. 2, 517-520.

7. J.D. Sally, Number of generators of ideals in local rings, Marcel Dekker (1978).

8. R.Y. Sharp, Steps in Commutative Algebra, Cambridge University Press 1990.

9. N.V. Trung, Bounds for the minimum numbers of generators of generalized Cohen-Macaulay ideals, J. of Algebra 90 (1984), 1-9.

10. O. Zariski and P. Samuel, Commutative algebra Vol. 2, Springer (1976). 